\title{
COLLISION DETECTION IN CLUTTERED DRIVING SCENES
}

Carissa M. Lemon \& George J. Andersen

University of California, Riverside

Riverside, California, USA

Email: clemo001@ucr.edu

\begin{abstract}
Summary: The purpose of the present experiment was to examine whether drivers' detection of collisions was altered when the driving scene was cluttered with scene objects. In this experiment stationary scene objects were manipulated by positioning them behind an approaching object and driver motion induced. We found that observers' collision detection performance (d') decreased with the presence of scene objects. These results indicate that the ability to detect a collision is altered by the presence of scene objects. In addition, performance was dependent on display duration, with greater sensitivity at increased durations. Moreover, the results showed a significant criterion shift between scene objects present and scene objects absent, with a decrease in identifying a collision object (hit rate) when scene objects were present but no difference in identification of a collision event when scene objects were absent. This suggests that the decreased performance was due to the inability to accurately determine a collision event because of apparent motion of background scene objects due to driver motion. Because the displays used in this experiment are akin to driving in a cluttered environment, the results of this research have important implications regarding driving safety and crash rates particularly in urban environments with complex scenes. Specifically, the results suggest that one factor in cluttered driving scenes is the apparent motion of background scene objects due to driver motion.
\end{abstract}

\section{INTRODUCTION}

An important driving task is the ability to detect an impending collision. Studies that have examined collision detection often present stimuli on linear trajectories or simulate motion on a linear path. Under these conditions a collision event is defined by the presence of expansion and constant bearing of the object. Constant bearing refers to an object maintaining an angular direction or fixed position in the optic flow field (Kaiser \& Mowafy, 1993). Non-collision objects on a linear path will have an angular direction that varies over time or a non-constant bearing.

Previous studies have examined the ability of observers to detect a collision event when multiple moving objects were present (Andersen \& Kim, 2001), and have assessed age-related differences in detecting collision events under stationary and moving observer conditions (Andersen \& Enriquez, 2006). Other studies have found a decreased ability to perceive movement in depth when moving objects are present (Gray, Macuga, \& Regan, 2004). Results of these studies, considered together, suggest that both expansion and bearing information are used in detecting a collision and the efficacy of these information sources may be reduced when objects are present in the scene. Driving environments usually do not consist of a single object approaching the driver. Rather, driving environments include other objects that are part of the driving scene 
(buildings, signs, parked vehicles, etc.) Under these conditions, the presence of static objects in the driving scene may introduce information that interferes with the ability to detect an impending object. Specifically, static objects in the scene will contain apparent motion due to the motion of the driver's vehicle through the environment. This observation suggests that collision detection may be impaired when static objects are present in the driving scene because the apparent motion of these objects may interfere with the ability to determine the bearing of an approaching collision object. The purpose of the present study was to examine this issue.

In the present study we examined collision detection performance for an object moving on a linear trajectory. The displays simulated an approaching spherical object in a 3-D scene. On half of the trials the approaching object would collide with the driver. On the other half of trials, the object would pass by the driver. The subjects were asked whether or not the object was on a collision path. We manipulated the display duration and presence of scene objects (Figure 1). If detection of an object on a collision path is affected by the apparent motion of surrounding scene objects, then we predict a decrease in sensitivity when scene objects are present as compared to when they are absent.

\section{METHOD}

\section{Subjects}

The subjects were 8 college-age students at the University of California, Riverside, who were paid for their participation. All the subjects had normal or corrected-to-normal vision and were naive as to the purpose of the experiment.

\section{Design}

The independent variables were display duration (1000ms, $3000 \mathrm{~ms}$, or $5000 \mathrm{~ms})$, collision event (collision or non-collision trajectory) and the presence of scene objects (objects present or objects absent), with a total of 450 trials. All the variables were run as within-subjects variables.

\section{Apparatus}

The displays were presented on a 58-inch $(148 \mathrm{~cm})$ flat screen plasma TV (Panasonic TH58PF12) with a pixel resolution of $1920 \times 1080$, controlled by a Windows 7 Professional Operating System on a Dell Precision T7500 workstation. The visual angle of the display was $42.28^{\circ}$. The refresh rate was $60 \mathrm{~Hz}$. The dimensions of the display on the monitor were $128.4 \mathrm{~cm}$ $(\mathrm{W}) \times 72.2 \mathrm{~cm}(\mathrm{H})$. Head position was fixed using a chinrest.

\section{Stimuli}

The stimuli were computer generated 3-D scenes of a roadway simulating forward motion. The ground surface on both sides of the roadway had a black and white checkerboard texture. The roadway had a width of 8 meters and the object was a bright red sphere ( 2 unit diameter or $0.65^{\circ}$ on the first frame). The approaching spherical object was initially positioned to the left or right 
of the roadway along an arc (approx. $20^{\circ}$ from the center of the display) at a fixed distance 115 units from the viewpoint. The object moved on a linear trajectory. Half of the trials consisted of motion trajectories that would collide with the driver. Collision events were created by crossing the trajectory, from the initial position, with the viewpoint of the driver. The total travel time from the initial position to the collision point was $7.2 \mathrm{sec}$. The remaining half of the trials consisted of motion trajectories that would pass by the driver. The 3-D velocity for collision and non-collision events was held constant. Non-collision events were created by altering the trajectory of a collision event by repositioning the endpoint to the left or right of the viewpoint from 4 to 6 units.

\section{Procedure}

The experiment was run in a darkened room. Subjects were informed that they would be shown a series of displays consisting of a 3-D scene, which simulated forward motion, along with a single sphere that was moving toward the driver. Their task was to determine whether the moving object would collide with them. The drivers were next shown a sequence of displays that demonstrated collision and non-collisions of the object. The demonstration displays depicted the complete trajectory. For non-collision events, the displays showed a distant object that approached and eventually passed to the left or right of the driver outside the field of view. After the drivers understood the task, they were presented with ten practice trials, half of which simulated a collision and half that simulated a non-collision, and were asked to indicate whether the object was on a collision trajectory. The practice trials were displayed the complete event. On a keyboard subjects pressed one button for non-collision responses and another button for collision responses. Feedback was not provided during the practice trials or experiment. As a means of ensuring that drivers understood the task and response, drivers were required to correctly identify nine of the ten practice trials before proceeding to the experimental trials.

\section{RESULTS}

The average proportions of hits (a collision response for trials that simulated a collision) and false alarms (a collision response for trials that did not simulate a collision) were calculated for each subject in each condition and were used to derive sensitivity (d') and response bias $(\beta)$ measures. Results of sensitivity were analyzed in a 3 (duration) $\times 2$ (scene objects' presence) analysis of variance (ANOVA). There was a main effect of scene objects $F(1,7)=13.360$, $\mathrm{p}=0.008$. These results indicate a decreased sensitivity in detecting a collision with the presence of scene objects. There was a main effect of duration $F(2,14)=21.444, \mathrm{p}<0.001$. These results indicate greater sensitivity in detecting a collision with increased duration. There was not a significant interaction of scene objects and display duration $F(2,14)=.747, \mathrm{p}=.492$. An analysis of $\beta$ indicated a significant criterion shift when scene objects were present. Analysis of false alarm rates showed that there was no significant difference in false alarm rate between scene objects present and scene objects absent $F(1,12)=.060, \mathrm{p}=.813$. However, there was a significant difference in hit rate $F(1,12)=15.821, \mathrm{p}=.005$, with hit rates decreasing when scene objects were present. 


\section{CONCLUSION}

We found a reduced sensitivity to detect a collision when scene objects were present. In addition, performance was dependent on display duration, with greater sensitivity at increased durations. That is, the apparent motion of surrounding scene objects alters a driver's ability to detect an object on a collision path and performance deteriorates at shorter durations. Given that previous research has shown a decrease in collision detection at higher speeds, a decrease in sensitivity when scene objects are present can be inferred as an additional risk in the ability to avoid an impending collision.

Furthermore, the results showed a significant criterion shift between scene objects present and scene objects absent. An analysis of hits (correctly identifying an impending collision as a collision) and false alarm (incorrectly identifying a non-collision event as a collision) rate indicated a decrease in hit rate when scene objects were present but no difference was found in hit rate when scene objects were absent. Considering that expansion information was readily available in both conditions, the shift in criterion provides support for the hypothesis that the presence of scene objects alters collision detection due to a misperception of bearing. Moreover, this particular shift in criterion has serious consequences in a real world driving scenario, with a reduced hit rate potentially leading to an increase in crash rate.

It should be noted that only linear object trajectories moving at a constant speed were examined in this study. Real world conditions can involve much more complex trajectories. The driver as well as the object can move in a linear or curved trajectory and both are often free to move at either a constant or varying speed. An important issue for future research will be to determine collision detection performance that involves more complex trajectories. For example, a future experiment could include collision objects that moves on curved trajectories and measures the effect of scene objects on these complex trajectories on collision detection performance.

Overall, the results of this research provide two important implications regarding driving safety and crash rates. First the presence of scene objects, as is typical in almost any driving scene, results in a reduced ability to accurately detect a collision object that has a fixed position in the visual field. An example of this would be an approaching vehicle on a collision path or object on the roadway. This suggests that in-vehicle technologies could be useful in cluttered driving environments to warn drivers of impending collisions, particularly when the driver's ability to detect a collision is reduced. A second implication concerns training young novice drivers. Previous research has shown that an impending collision of an object on a linear trajectory is specified, in part, by the object maintaining a fixed position or bearing in the visual field. The present findings suggest that the decreased performance in detecting collisions in cluttered environments is due to a reduced ability to determine bearing information of the object on a collision path. One possible approach to improve driver safety would be to develop training procedures to improve detection performance when objects are present in the driving scene by training drivers to detect a constant bearing of an approaching object. Studies have shown that training with near threshold stimuli can improve visual performance for younger and older adults (Andersen, Ni, Bower \& Watanabe, 2010). Such techniques could be used to improve the speed and accuracy of collision detection when apparent motion of background scene objects are present, with the expectation that greater sensitivity to detect constant bearing would aid in 
detecting a collision event and increase the speed of detection. Increasing the speed of detection would result in more time to execute collision avoidance maneuvers resulting in increased driver safety. Such training programs could be especially useful as part of the licensure for young novice drivers, who have a high rate of vehicular crashes (Mayhew \& Simpson, 2002).

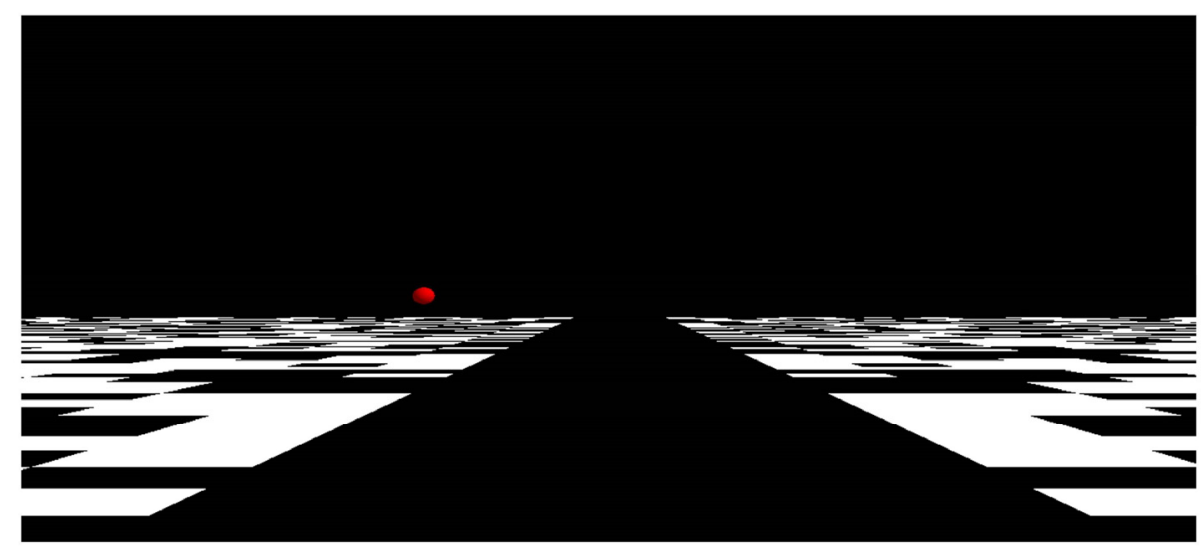

Figure 1a. Scene objects absent

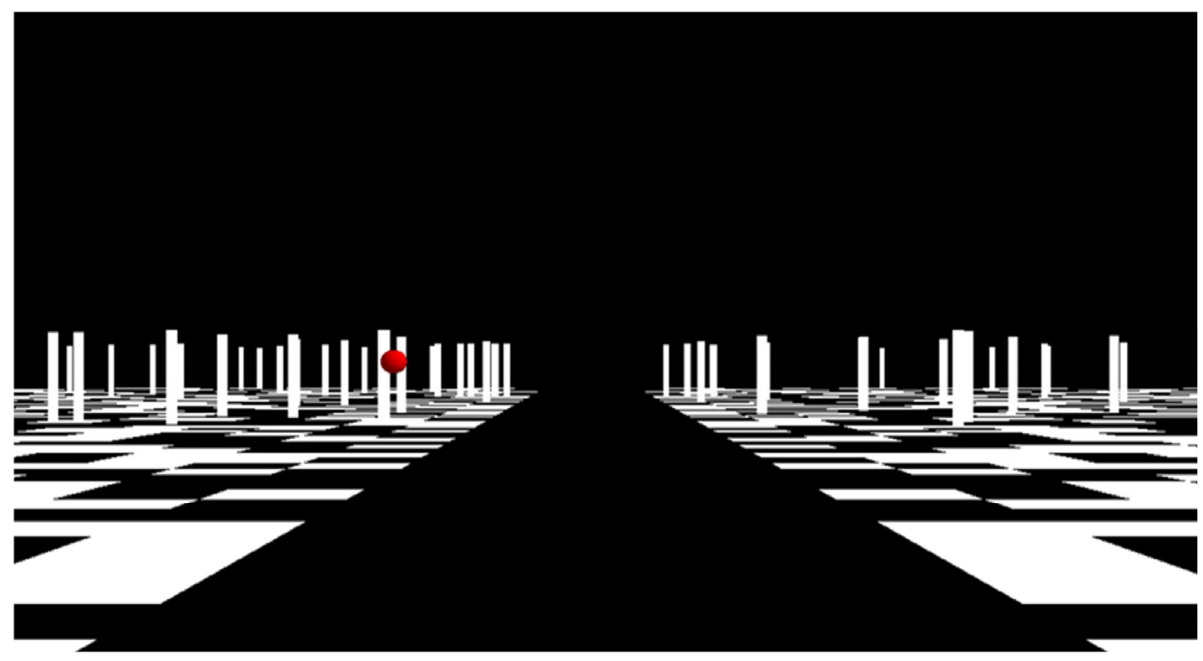

Figure 1b. Scene objects present

Figure 1. Example of stimuli. Figure 1a is an instance of the scene objects absent condition. Figure $1 \mathrm{~b}$ is an example of the scene objects present condition 


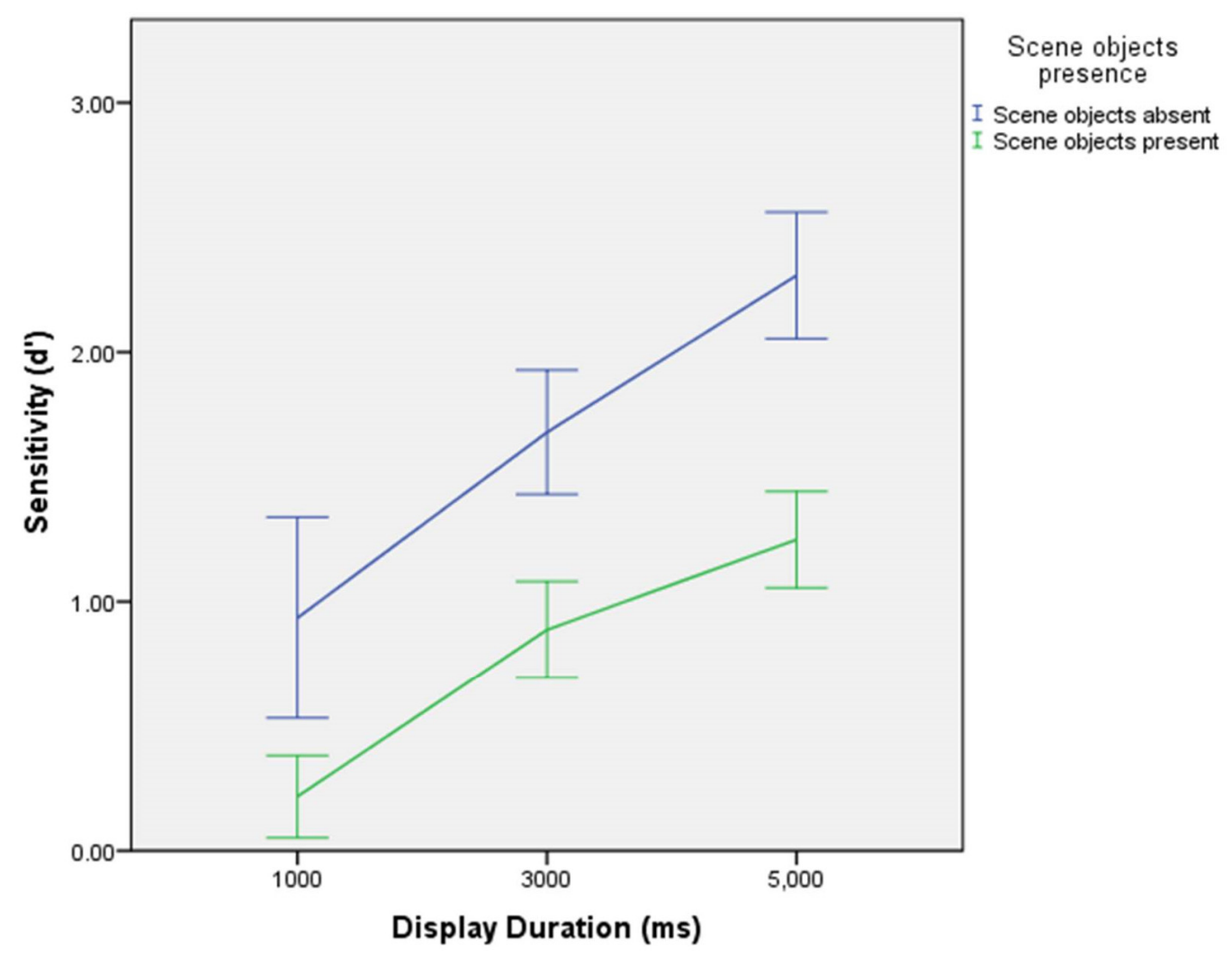

Figure 2. Results

Figure 2. Results for Sensitivity (d') from experiment. The results for d' when scene objects were absent and when scene objects were present, as a function of display duration. Errors bars represent standard error (+/- $1 \mathrm{SE})$.

\section{REFERENCES}

Andersen, G. J., \& Enriquez, A. (2006). Use of landmarks and allocentric reference frames for the control of locomotion. Visual Cognition, 13(1), 119-128.

Andersen, G. J., \& Kim, R. D. (2001). Perceptual information and attentional constraints in visual search of collision events. Journal of Experimental Psychology: Human Perception and Performance, 27(5), 1039.

Andersen, G. J., Ni, R., Bower, J. D., \& Watanabe, T. (2010). Perceptual learning, aging, and improved visual performance in early stages of visual processing. Journal of vision, $10(13), 4$. 
Gray, R., Macuga, K., \& Regan, D. (2004). Long range interactions between object-motion and self-motion in the perception of movement in depth. Vision Research, 44, 179-195.

Kaiser, M. K., \& Mowafy, L. (1993). Optical specification of time-to- passage: Observers' sensitivity to global tau. Journal of Experimental Psychology: Human Perception and Performance, 19, 1028-1040.

Mayhew, D. R., \& Simpson, H. W. (2002). The safety value of driver education and training Injury Prevention, 8, 3-8. 